\title{
Editorial
}

\section{CREACIÓN DEL PROGRAMA DE PROCURACIÓN Y DONACIÓN DE CUERPOS DE LA UNIVERSIDAD NACIONAL DE CÓRDOBA - ARGENTINA}

\author{
Susana N. Biasutto \\ Programa de Procuración y Donación de Cuerpos, Facultad de Ciencias Médicas, \\ Universidad Nacional de Córdoba, Córdoba, Argentina
}

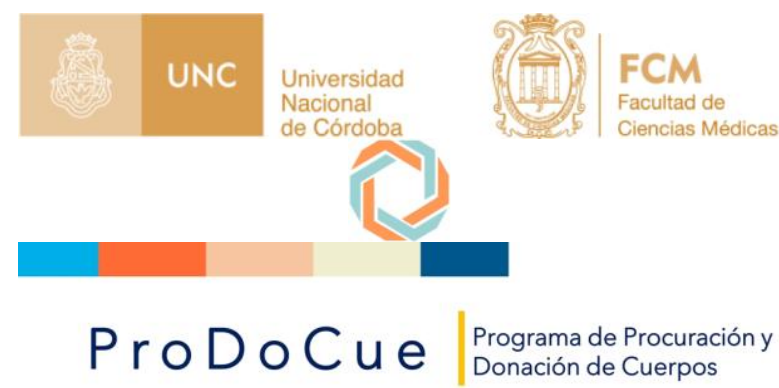

La necesidad de material cadavérico y de la disección, para la docencia-aprendizaje de la Anatomía Normal, no se encuentra en discusión a nivel mundial, aún disponiendo de los recursos tecnológicos más avanzados y sofisticados. Son muchos los estudios científicos que avalan la importancia de este recurso para la formación médica y las diferencias significativas resultantes de su carencia. (Ashraf-Aziz et al., 2002; Drake et al., 2002; Miller et all, 2002; Arráez-Aybar et al., 2004; Biasutto et al., 2006, Azer y Eizemberg, 2007; Halou et al., 2013; Biasutto et al, 2014a; Narváez-Hernández y Murillo-Rábago, 2014; Burgess y Ramsey-Stewart, 2015; Kramer y Hutchinson, 2015).

En la actualidad, la utilización de cuerpos no reclamados (como se hacía antiguamente) no es bien considerada por la Federación Internacional de Asociaciones de Anatomistas (2017) y se contrapone a los Derechos Humanos de las personas.

La vía para suplir esta necesidad no resulta novedosa, pero sí compleja.

Son muchos los países, en los diferentes continentes, que cuentan con programas y normas para la donación de cuerpos destinados a docencia e investigación universitaria. El mapa de distribución muestra una significativa deficiencia en Sudamérica, incluso en la comparación con Asia y África (Cornwall y Stringer, 2009; Anyanwu y Obikili, 2012; Riederer et al, 2012; Anyanwu et al, 2014; Biasutto et al, 2014 a, b; Kramer y Hutchinson, 2015, Habicht et al, 2018). Desde hace más de 40 años algunos países de Europa y Canadá cuentan con modernos programas y/o leyes para la Procuración y Donación de Cuerpos y Tejidos para Docencia e Investigación universitaria (Riederer et al, 2012; McKlea y Stringer, 2013; Biasutto et al, 2014a). Las cátedras o centros de Anatomía de esos países son los que disponen de cuerpos más que suficientes para los requisitos del pre y postgrado; que pueden ser selectivos y aún rechazar donaciones.

Sudamérica (a excepción de Uruguay) es la región con mayor atraso en el desarrollo de políticas en este sentido. Aunque Brasil y Chile han elaborado algunos programas locales (no siempre exitosos), no han logrado aún superar la dificultad causada por la carencia de cuerpos (da Rocha et al, 2013; Biasutto et al., 2014a; Biasutto et al, 2018; Habicht et al, 2018).

Argentina carece de una ley específica para la donación de cuerpos, y a diferencia de los programas para donación de órganos para trasplante, la donación de cuerpos se basa en programas locales, en torno a una universidad, a la cual se realizará la donación.

La necesidad de las Cátedras de Anatomía es inobjetable, pero consideramos que no hay procedimientos rápidos para abordar un tema tan sensible y de relativa complejidad (Garay et al., 2015).

Luego de muchos años y dificultades, en 2018 propusimos un proyecto de investigación que, 
con rigor científico, aportara información sobre las características generales y socio-culturales de las diversas poblaciones que estarían involucradas en un programa de esta naturaleza, y que a su vez instale la consideración del tema progresivamente desde poblaciones más cercanas a la problemática, para luego abordar aquellas más distantes. El proyecto fue aprobado por al Secretaría de Ciencia y Tecnología de la Universidad Nacional de Córdoba para el período 2018-2020.

El desarrollo del proyecto permitió la formación de recursos humanos no solo en investigación, sino además con capacidad de informar y orientar para la procuración y donación de cuerpos.

Los resultados del estudio fueron sorprendentes aún para las mejores expectativas previas (Biasutto et al., 2019a, 2019b, 2019c, 2020, 2021). El reconocimiento de la importancia de los cuerpos para la docencia-aprendizaje de la Anatomía por estudiantes y profesionales de la salud, y la voluntad para donar el propio cuerpo de estos grupos y de la población general de la ciudad de Córdoba como contribución a la docencia y la investigación permitieron fundamentar la propuesta para la creación del Programa de Procuración y Donación de Cuerpos de la Facultad de Ciencias Médicas de la Universidad Nacional de Córdoba.

Así, el 22 de abril de 2021, el H. Consejo Directivo de la Facultad aprobó la creación del Programa y la constitución de un Comité que hiciera cumplir las normas éticas y organizara las actividades tendientes a dar cumplimiento a su finalidad.

Con un grupo de docentes y un importante número de ayudantes-alumnos de Anatomía Normal estamos afrontando el desafío de cambiar el rumbo en la donación de cuerpos en Argentina, confiados en que la generosidad y solidaridad de la población de Córdoba será un ejemplo para el país.

\section{Bibliografía}

Anyanwu EG, Obikili EN. 2012. Dissecting the dissectors: knowledge, attitude, and practice of body bequests by Nigerian anatomists. Anat Sci Educ. 5: 347-53. doi: 10.1002/ase.1298.

Anyanwu EG, Obikili EN, Agu AU. 2014. The Dissection Room Experience: A Factor in the Choice of Organ and Whole Body Donation - A Nigerian Survey. Anat Sci Educ 7: 56-63.

Arraéz-Aybar LA, Castaño-Collado G, Casado Morales MI. 2004. Dissection from the Spanish anatomist's perspective: Aims, attitude and related aspects. Rec. Anat. 281B: 15-20.
Ashraf Aziz M, Mckenzie JC, Wilson JS, Cowie RJ, Ayeni SA, Dunn BK. 2002. The human cadaver in the age of biomedical informatics. Anat. Rec. 269: 20-32.

Azer SA, Eizenberg N. 2007. Do we need dissection in an integrated problem-based learning medical course? Perceptions of firstand second-year students. Surg Radiol Anat. 29:173-80

Biasutto SN, Caussa LI, Criado del Río LE. 2006. Teaching Anatomy: Cadavers vs. Computers. Annals of Anatomy 188: 187-90

Biasutto SN, David OP, Spinelli MA, Urrutia D, Bertocchi Valle AJ, Weigandt DM, Molina Vargas IE, Vargas RAA, Navarro LM, Siemsen $S G$, Turri F, Longoni N, De Oro F, Prost SA. 2019c. Research on body donation willingness in Cordoba-Argentina: medical and dentist doctors' attitude. Rev Arg de Anat Clin 11: 107 14.

Biasutto SN, Garay MB, Rives MV, Uanini $F$, Albrecht A, Ortiz LB, Gerbaldo MV. 2018a. La percepción de los estudiantes de primer año de Medicina en la sala de disección y su incidencia sobre la procuración de cuerpos. Rev Arg de Anat Clin 10: 44-51.

Biasutto SN, Molina Vargas IE, Weigandt DM, Mora MV, Vargas RAA, Bertocchi Valle AJ, Uanini MF, David OP, Urrutia D, Spinelli MA, Olivera MNJ, Gimenez M, Nefa Salera $M$, Decouvette A, Ferrero LJ. 2019a. Reactions of first year medical students in the dissection room, with prosected corpses, and the incidence on own body donation. Rev Arg de Anat Clin 11: 18-29

Biasutto SN, Navarro LM, Turri F, Longoni N, Bertocchi Valle AJ, Siemsen SG, De Oro $F$, Molina Vargas IE, David OP, Spinelli MA, Urrutia D. 2020. Diferencia entre médicos y odontólogos respecto a la donación del propio cuerpo para docencia e investigación en Córdoba - Argentina. Rev Arg de Anat Clin 12: 17-22.

Biasutto SN, Navarro LM, Siemsen SG, Turri $F$, Longoni N, Bertocchi Valle AJ, David OP, Spinelli MA, Urrutia $D$, Weigandt DM, De Oro $F$, Molina Vargas IE. 2021. Voluntad para la donación del cuerpo en la población de Córdoba en Argentina. Rev Arg de Anat Clin 12: 17-25.

Biasutto SN, Sharma N, Weiglein AH, Martinez Benia F, McBride J, Bueno-López JL, Kramer $K$, Blyth $P$, Barros MD, Ashiru O, Ballesteros LE, Moxham BJ, Krishnan S. 2014a. Human Bodies to Teach Anatomy: Importance and Procurement - Experience with Cadaver Donation. Rev Arg de Anat Clin 6 (2): 72-86

Biasutto SN, Sharma NA, McBride J, Krishnan S, Vatsalaswamy P, Garud RS, Kharat VS, du Toit 
DF, Redwood C, Fisk W, Townsend G, Piplani $M L$, Romero-Reverón $R$, Rae G, Kachlik $D$, Barut C, Khayrullin RM. 2014b. Part II - Human Bodies to Teach Anatomy: Importance and Procurement - Experience with Cadaver Donation. Rev Arg de Anat Clin 2014, 6 (3): 163-175

Biasutto SN, Spinelli MA, Weigandt DM, Mora MV, Bertocchi Valle AJ, Vargas RAA, Molina Vargas IE, David OP, Urrutia D. 2019b. Attitude of medical students to body donation in the National University of Cordoba - Argentina. Rev Arg de Anat Clin 11: 70-83.

Burgess A, Ramsey-Stewart G. 2015. Anatomy by whole body dissection: a focus group study of students' learning experience. Adv Med Educ Pract. 6: 533-37.

Cornwall J, Stringer MD. 2009. The wider importance of cadavers: Educational and research diversity from a Body Bequest Program. Anat Sci Educ 2: 234-37.

da Rocha AO, Tormes DA, Lehmann N, Schwab $R S$, Teixeira Canto R. 2013. The body donation program at the Federal University of Health Sciences of Porto Alegre: A successful experience in Brazil. Anat Sci Educ 6: 199-204.

Drake RL, Lowrie DJ (jr), Prewitt CM. 2002. Survey of gross anatomy, microscopic anatomy, neuroscience, and embryology courses in medical school curricula in the United States. Anat. Rec. 269: 118-22.

Garay MB, Albrecht A, Rives MV, Ortiz L, Uanini F, Gerbaldo V, Biasutto SN. 2015. Perception of first year medical students in relation with the cadaver and the dissection room. Rev Arg de Anat Clin 7: 213.

Habicht JL, Kiessling C, Winkelmann A. 2018. Bodies for Anatomy education in medical schools: An overview of the sources of cadavers worldwide. Aceptado para publicación en Academic Medicine DOI: 10.1097/ACM. 0000000000002227.

Halou H, Chalkias A, Mystrioti D, lacovidou N, Vasileiou PVS, Xanthos T. 2013. Evaluation of the willingness for cadaveric donation in Greece. A population-based study. Anat Sci Educ 6: 48-55. DOI: 10.1002/ase.1304

International Federation of Associations of Anatomists. 2017. Recommendations of good practice for the donation and study of human bodies and tissues for anatomical examination. http://www.ifaa.net/wp-content/uploads/2017/ 09/IFAA-guidelines-220811.pdf (11/07/21).

Kramer B, Hutchinson EF. 2015. Transformation of a cadaver population: Analysis of a South African cadaver program, 1921-2013. Anat Sci Educ. 8: 445-51. DOI: 10.1002/ase.

McKlea K, Stringer MD. 2013. Why do potential body donors decide against donating?. N Z Med J. 126: 51-58.

Miller SA, Perrotti W, Silverthorn DU, Dalley AF, Rarey KE. 2002. From college to clinic: Reasoning over memorization is key for understanding anatomy. Anat. Rec. 269: 69-80.

Narváez-Hernández E, Murillo-Rábago El. 2014. Herramientas de estudio utilizadas por alumnos de ciencias de la salud en la materia de Anatomía. Inv Ed Med 3: 204-08

Riederer BM, Bolt S, Brenner E, Bueno-López JL, Circulescu ARM, Davies DC, De Caro R, Gerrits PO, McHanwell S, Pais D, Paulsen F, Plaisant O, Sendemir E, Stabile I, Moxham B. 2012. The legal and ethical framework governing Body Donation in Europe - 1st update on current practice. Eur J Anat 16: 1-21. 